\title{
The EPIC-InterAct Study: A Study of the Interplay between Genetic and Lifestyle Behavioral Factors on the Risk of Type 2 Diabetes in European Populations
}

\author{
Nita G. Forouhi • Nicholas J. Wareham
}

Published online: 11 October 2014

(C) The Author(s) 2014. This article is published with open access at Springerlink.com

\begin{abstract}
The rising prevalence of type 2 diabetes around the world and the global pattern of variation in risk between countries have been widely attributed to an interplay between rising rates of obesity and poor lifestyles, and genetic or developmental susceptibility to disease. Although this general hypothesis has been in existence for more than 50 years, the precise mechanisms that may explain it have remained uncertain. Advances in technology and the application of new methods in large scale population studies have made it possible to study these mechanisms. The InterAct project, funded by the European Commission, is a large case-cohort study which has verified 12,403 incident cases of type 2 diabetes, facilitating the study of genetic and lifestyle factors on the risk of type 2 diabetes among European populations.
\end{abstract}

Keywords Type 2 diabetes - Prevalence - Diet - Physical activity · Nutritional biomarker · Aetiology · Prevention · Genetic factors · Gene-lifestyle interaction

\section{Introduction}

Type 2 diabetes is a major contemporary public health issue because of its high and rising prevalence worldwide and its association with diverse complications that lead to premature morbidity and mortality. The cost of the disease to individuals, carers, health systems, and society is considerable. The latest estimates from the International Diabetes Federation suggest

N. G. Forouhi $(\square) \cdot$ N. J. Wareham

Medical Research Council Epidemiology Unit, University of

Cambridge School of Clinical Medicine, Institute of Metabolic

Science, Cambridge Biomedical Campus, Cambridge CB2 0QQ, UK

e-mail: nita.forouhi@mrc-epid.cam.ac.uk

N. J. Wareham

e-mail: nick.wareham@mrc-epid.cam.ac.uk that 382 million people had diabetes in 2013, and that this prevalence is projected to increase to 592 million by 2035 [1•].

Several randomised controlled trials have provided strong evidence that the primary prevention of type 2 diabetes is possible through lifestyle interventions aimed at changing diet and physical activity [2-6]. However, many uncertainties remain about how to translate this potential into action in real-world settings. Firstly, it is unclear how to determine the appropriate balance between investment in approaches to prevention that focus on individuals at high risk of diabetes as opposed to efforts that seek to impact on the entire population. Secondly, where high risk approaches are proposed, it is unclear whether detailed assessment of individual susceptibility to diabetes through estimation of genetic risk, for example, plays a role in identifying people who may benefit from individualised prevention. Finally there are uncertainties about the details of the nutritional and dietary guidelines that should form the basis of public health targets as opposed to recommendations for change among individuals at high risk. When the World Health Organization reviewed the evidence for the association between diet and the development of type 2 diabetes a decade ago, no dietary factor fulfilled the criteria to qualify for convincing grade evidence [7]. Progress has been made since then with evidence arising predominantly from US-based cohorts, but systematic, large-scale evidence from Europe-based populations was still unavailable. Moreover, the investigation of an interaction between genetic and lifestyle factors has been a challenge as this would require an adequately powered study with a large sample size, availability of genetic data as well as robustly collected valid data on lifestyle factors.

To address these research gaps, the InterAct project (http:// www.inter-act.eu) was funded by the European Union (EU) within its FP6 framework to investigate the interaction of genes and lifestyle on the risk of type 2 diabetes. A feature of this study that made it suitable for this purpose was that it 
was embedded in a multi-country cohort study (The EPIC study) with heterogeneity of dietary exposures which were assessed within a standardised framework. The initial study was very large and with sufficient elapsed time so that InterAct was able to accrue four million person-years of follow-up and ascertain and verify 12,403 incident cases of type 2 diabetes. Previous studies on gene-lifestyle interaction have demonstrated the importance of correctly parameterising and characterising the main lifestyle exposures before proceeding to investigate interaction [8]. Therefore, a key initial stage in InterAct was the examination of the nature and magnitude of the relationship between a variety of lifestyle behavioural factors and incident diabetes. Not only did this process set the stage for the examination of interaction, but it is also of public health importance in its own right as it guides the design of public health interventions.

\section{The InterAct Project}

The InterAct project is co-ordinated by the Medical Research Council Epidemiology Unit, Cambridge, and it is largely centred on constructing a nested case-cohort study within eight of the ten participating countries of the European Prospective Investigation into Cancer (EPIC) cohort study: France, Italy, Spain, UK, the Netherlands, Germany, Sweden, and Denmark. Collaborators in 26 European research centres ascertained and verified 12,403 cases of incident type 2 diabetes from a total cohort of 340,234 people with four million person-years of follow-up, and randomly selected 16,835

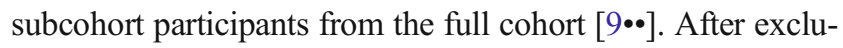
sions, a representative subcohort of 16,154 individuals remained. A design feature of the case-cohort design is that due to the process of random selection, a number of future disease cases are also included within the subcohort. Thus, the total sample of EPIC-InterAct included 12,403 case participants with type 2 diabetes, and 16,154 subcohort participants, of whom 778 formed an overlap group between the casegroup and the subcohort group as they had incident type 2 diabetes during follow-up. The case-cohort design should be thought of as a subcohort study with additional incident cases added rather than as a case-control study. The statistical analysis for the case-cohort design accounts for the small number of cases in the subcohort. Incident type 2 diabetes cases were ascertained up until 31 December 2007 through a review of several sources of evidence including self-report, linkage to primary care registers, secondary care registers, medication use, hospital admissions and mortality data. No diabetes cases were ascertained solely by self-report, but rather confirmatory evidence was sought for all cases with information about incident type 2 diabetes with fewer than two independent sources, including a review of individual medical records in some centres $[9 \bullet \cdot$.

\section{The Association Between Diet, Nutrition, and the Risk of Type 2 Diabetes}

Detailed self-report assessments of food intake were made in each participating centre using validated country-specific dietary questionnaires, mostly food frequency questionnaires (FFQ). A bespoke European nutrient database was used [10]. Comprehensive approaches to analysis were taken, including adjustment for energy intake, statistical models that included the residual method where relevant, measurement error correction using calibration of FFQ data against the 24-h recall in a subset of the sample, and adjustment for a range of potential confounding factors including known diabetes risk factors, demographic, social, lifestyle and dietary factors.

\section{Food Groups and the Development of Type 2 Diabetes}

The publication of three meta-analyses had indicated a positive association between a diet rich in red and processed meat consumption and the risk of type 2 diabetes [11-13], with a focus largely on US-based studies. Though individual studies investigating this had started emerging from Europe [14-16], there remained a lack of a systematic approach and adequate sample size. EPIC-InterAct reported that across eight European countries, for every 50 -g increase in consumption of red meat and processed meat, there was a higher risk of future type 2 diabetes, with a hazard ratio (HR) of 1.08 [95\% confidence interval (CI) 1.03, 1.13] and 1.12 (95\%CI: 1.05, 1.19), respectively, independent of a comprehensive range of potential confounding factors [17].

In contrast to the more consistent findings for meat intake and diabetes risk, there were previously inconclusive findings for the association between the consumption of dairy products and diabetes risk, in particular for dairy sub-types [18]. There was a general paucity of such research in Europe, which was surprising given that Europe has the highest intake of dairy products in the world [19]. A previous analysis in the EPIC study had shown substantial variation in the amount of total and types of dairy products consumed across Europe, with high cheese consumption in France and high yoghurt consumption in Sweden and the Netherlands [20]. With its varying dairy products intake, the EPIC-InterAct study reported that intake of total dairy products, or of milk, was not related to future diabetes risk, but the consumption of combined fermented dairy products (yoghurt; cheese; thick fermented milk) was associated with a reduced risk of incident diabetes (HR 0.88, $95 \%$ CI 0.78, 0.99) [21].

Evidence on the role of fish intake for diabetes risk has been conflicting, ranging from proposed beneficial [22] to harmful [23, 24] to null effects [25]. Findings from the EPIC-InterAct study have indicated that only sub-types of fish intake, rather than total fish intake, may be related to diabetes risk [26]. Further to the InterAct findings, evidence 
is accumulating that geographic location plays an important role in the nature of the association between fish intake and diabetes risk $[25,27 \bullet]$.

Fruit and vegetable intake is a recommended component of a healthy diet, but little evidence was available generally, and particularly from Europe, for a link between intake and diabetes risk [28]. We conducted a systematic review of previously published literature and then performed a meta-analysis along with new data from EPIC-InterAct. Our analysis of 179,956 participants and 19,123 incident type 2 diabetes cases reported that those consuming the highest fruit and vegetable intake had a $7 \%$ (95 \% CI: $0.87-1.00)$ lower risk of diabetes when compared with the lowest intake consumers, with a particular benefit for consumption of green leafy vegetables [29]. This work has provided more convincing evidence for the benefits of fruit and vegetable consumption for the prevention of diabetes.

\section{Beverages and the Development of Type 2 Diabetes}

There has been increasing interest in associations between the consumption of sweetened beverages and the risk of developing diabetes, but data from Europe had been sparse, with only one European country (Finland) contributing to a systematic review and meta-analysis [30 ]. EPIC-InterAct investigated associations between both sugar-sweetened beverage (SSB) and artificially sweetened beverage (ASB) intake and the development of incident type 2 diabetes [31•]. In adjusted analyses, EPIC-InterAct reported a multivariable-adjusted $22 \%$ (95\% CI: $9 \%$ to $38 \%$ ) higher incidence of type 2 diabetes for every one serving/day (336 g standard size can of drink) of greater habitual consumption of SSB. A previous meta-analysis was unable to distinguish associations with and without adjustment for obesity [32], but in EPIC-InterAct there was a persisting $18 \%$ higher incidence of diabetes $(95 \% \mathrm{CI}$ : $6 \%$ to $32 \%$ ) even after adjustment for adiposity. Though there was a positive association between habitual ASB consumption and diabetes risk, the association became non-significant after accounting for baseline obesity (body mass index); HR of 1.11 $(95 \%$ CI: $0.95,1.31)$ for every one serving greater habitual consumption of ASB, in adjusted analyses including adjustment for energy intake and body mass index [31•].

EPIC-InterAct also reported that greater tea consumption was associated with a lower risk of type 2 diabetes among Europeans. Individuals who reported drinking at least four cups of tea per day had a $16 \%$ (95\% CI: $0.71-1.00)$ lower risk of developing type 2 diabetes compared to those who reported no tea consumption [33].

\section{Dietary Patterns and the Development of Type 2 Diabetes}

There has been increasing recognition that people consume overall diets within certain patterns which are largely healthy or unhealthy [34]. Therefore, it is of interest to study the association of dietary patterns with health, in addition to the associations of individual food groups and foods. In the first large-scale study, EPIC-InterAct has reported that people who ate a dietary pattern in concordance with the principles of the Mediterranean diet had a reduced risk of developing diabetes [35]. The InterAct Consortium further extended its enquiry to include other predefined dietary patterns and also applied reduced rank regression (RRR) as a mixture of a hypothesisoriented and an exploratory approach that is aimed at identifying food group combinations that explain a maximum of variation in (disease-related) response variables [36]. In EPICInterAct there was no association between the alternative Healthy Eating Index (aHEI) and the Dietary Approaches to Stop Hypertension (DASH) score and diabetes risk, but inverse associations were observed for three RRR-derived dietary pattern scores [37]. The study confirmed that adherence to specific dietary patterns, commonly characterised by high intake of fruits or vegetables and low intake of processed meat, sugar-sweetened beverages, and refined grains, may lower type 2 diabetes risk.

Nutrients, Nutritional Compounds, and the Development of Type 2 Diabetes

Past research on the association between protein intake and risk of diabetes included the limitations of small sample size or cross-sectional evidence [38, 39], lack of investigation of type of protein (animal or plant origin) [40] or lack of association in obesity-adjusted analyses [41]. In EPIC-InterAct the incidence of type 2 diabetes was higher in those with the highest intake of total protein [per $10 \mathrm{~g}$ : HR: 1.06 (95\% CI: $1.02,1.09), p$-trend $<0.001]$ and animal protein [per $10 \mathrm{~g}$ : HR: $1.05(1.02,1.08), p$-trend $=0.001]$, but in contrast, intake of plant protein was not associated with type 2 diabetes [per $10 \mathrm{~g}$ : HR: 1.04 (95 \% CI: 0.93, 1.16), $p$-trend=0.098] [42], after adjustment for known confounders. These findings are consistent with advice to limit diets high in dietary proteins, particularly from animal sources.

EPIC-InterAct also investigated carbohydrates intake, together with their glycaemic properties, as expressed by the glycaemic index (GI) and the glycaemic load (GL), and found no association with diabetes risk [43]. This is in contrast to findings from three meta-analyses of prospective, predominantly American studies, published prior to InterAct findings, that reported increased diabetes risk in the highest GI and GL categories, ranging from an increased risk of $16 \%(\mathrm{GI})$ and $20 \%$ (GL) to $58 \%$ (both GI and GL) [44-46]. Further reports of a positive association between both GI and GL and risk of incident type 2 diabetes subsequent to the publication of findings from InterAct are also at variance with the null InterAct finding [47]. The investigation of GI values across different countries or even within the same population (such as one of the participating cohorts in InterAct) highlighted an 
important issue that an expansion of GI tables and systematic GI value assignment to foods is needed to improve the validity of GI values in cross-study comparisons. Analyses on the association between fibre intake and diabetes risk are complete in InterAct, and will be published combined with an updated systematic review and meta-analysis in the near future.

EPIC-InterAct found no association between dietary energy density of solid and semi-solid foods and incident type 2 diabetes [48]. This work highlighted the complexities of consensus on how energy density should be calculated, the potential role of under- or over-reporting, and the challenges of teasing out direct (on T2D) versus indirect (on obesity, weight gain) effects of dietary energy density, but concluded that energy density was likely to have only a small influence on the development of $\mathrm{T} 2 \mathrm{D}$ even if these complexities were overcome.

There has been interest in the potential effects of flavonoids, a group of nutritional compounds of the polyphenol family that are contained in plant-based foods such as fruits, vegetables, nuts, legumes, cocoa, some cereals, and in tea, wine, and juice, but past evidence has been inconclusive for association with diabetes [49-54]. EPIC-InterAct reported that total flavonoid intake tended towards an inverse association with incident diabetes (HR 0.90, 0.77, 1.04, $p$-trend= 0.04 ), but higher and stronger significant inverse associations were found for flavonoid sub-classes [55]. In particular, the HR when comparing the top quintile versus the bottom quintile of intake of flavonols were $0.81(0.69,0.95)$, and flavanols $0.82(0.68,0.99)$, including flavan-3-monomers $0.73(0.57$, 0.93 ) demonstrated an association with reduced incident diabetes. Further inspection of individual flavonoids (beyond classes of flavonoids) highlighted significant inverse trends between intakes of all individual flavan-3-ol monomers and diabetes incidence [56]. Additionally, proanthocyanidin dimers and trimers, but not proanthocyanidins with a greater degree of polymerisation were inversely associated. Among the flavonol sub-class, myricetin (HR 0.77, 0.64, 0.93) was associated with a lower incidence of T2D. Our findings provided the first comprehensive examination of both flavonoid classes and individual flavonoids, using a comprehensive flavonoid food composition database that was developed using both the Phenol-Explorer (the U.K. Food Standards Agency) and the U.S. Department of Agriculture databases [55]. These findings provide meaningful contributions toward our understanding of how a dietary pattern rich in plant-based foods helps with the prevention of type 2 diabetes.

In the context of strong epidemiological evidence that circulating concentrations of 25-hydroxy vitamin D $[25(\mathrm{OH}) \mathrm{D}]$ are related inversely to the development of type 2 diabetes [57], EPIC-InterAct also appraised the role of dietary vitamin $\mathrm{D}$, as a contributing source of circulating $25(\mathrm{OH}) \mathrm{D}$ levels. The study reported a null association between vitamin $\mathrm{D}$ intake and risk of diabetes in both the total and sex-specific analyses. This should be interpreted in light of the relatively greater contribution of endogenous synthesis of $25(\mathrm{OH}) \mathrm{D}$ following sunlight exposure, and thus the evidence from our large-scale study confirming no association of type 2 diabetes with dietary vitamin $\mathrm{D}$ intake provides important information in helping to distinguish between dietary and endogenously synthesised vitamin D.

\section{Moving Beyond Self-Report Assessment of Diet to Using Nutritional Biomarkers}

To overcome the well-acknowledged limitation of measurement error in nutritional epidemiology, InterAct has invested substantial effort into generating evidence on blood-based objective biomarkers that reflect nutritional intake.

There has been little comprehensive assessment of objectively measured circulating fatty acids in relation to diabetes risk across the range of individual fatty acids of varying carbon chain lengths. Reasons include the expense of measurement and the challenge of measuring them in large studies due to the substantial laboratory run-time and personnel time needed to process fatty acids on a large scale. In a collaboration between analytical chemists and epidemiologists, InterAct undertook to establish a high throughput method to measure 37 fatty acids in the plasma phospholipid fraction at the MRC Human Nutrition Research, Cambridge [58]. This enabled the measurement of plasma phospholipid fatty acids on an unprecedented scale, allowing the measurement of nearly 28,000 EPIC-InterAct samples.

In analyses focused on nine individual saturated fatty acids (SFAs), there were opposite directions of association with diabetes with SFA of varying carbon chain lengths [59•]. As shown in Fig. 1, odd-chain SFAs were associated inversely with incident diabetes [HR $(95 \% \mathrm{CI})$ per $1 \mathrm{SD}$ difference for 15:0 (pentadecanoic acid) $0.79(0.73,0.85)$, and for 17:0 (heptadecanoic acid) $0.67(0.67,0.71)]$, while evenchain SFAs were positively associated [14:0 (myristic acid) HR 1.15 (1.09, 1.22), 16:0 (palmitic acid) HR 1.26 $(1.15,1.37)$, and 18:0 (stearic acid) $1.06(1.00,1.13)]$. The very-long chain SFA (number of carbon atoms in chains varying between 20 and 24) were associated with lower diabetes incidence (HR ranging between 0.72 and 0.81 per 1 SD difference). The findings for odd-chain SFA can be understood in terms of their exogenous source from dairy fat [60-62] as well as the positive correlations in EPIC-InterAct between dairy products and the sum of pentadecanoic (15:0) and heptadecanoic (17:0) acids. This is in line with recent work, including from EPIC-InterAct, indicating that dairy products consumption is associated with lower risk of diabetes $[18,21,63 \bullet]$. The findings for even-chain SFA are more 




Fig. 1 Hazard ratios and $95 \%$ CIs for associations between plasma phospholipid saturated fatty acids and incident type 2 diabetes in EPICInterAct. Associations per $1 \mathrm{SD}$ difference in (A) even-chain fatty acids (saturated fatty acid [SFA] group 1: the sum of 14:0, 16:0, and 18:0), (B) odd-chain fatty acids (SFA group 2: the sum of 15:0 and 17:0), and (C) long- and very-long-chain fatty acids (SFA group 3: the sum of 20:0, 22:0, 23:0, and 24:0) and type 2 diabetes. Estimates are per country and the pooled estimate is based on random-effects meta-analysis. The analyses included 12132 cases of type 2 diabetes and 15919 people in the subcohort (including 755 individuals with type 2 diabetes in the subcohort); used age as the underlying time variable; and were adjusted for centre, sex, smoking status, alcohol intake, physical activity, education level, total energy intake, and BMI. Reproduced from Forouhi NG, Koulman A, Sharp SJ, et al. Differences in the prospective association between individual plasma phospholipid saturated fatty acids and incident type 2 diabetes: the EPIC-InterAct case-cohort study. The Lancet Diabetes \& Endocrinology. 2014; pii: S2213-8587(14)70146-9, with permission from Elsevier complex as these SFA appear to be mainly derived from hepatic endogenous synthesis (de novo lipogenesis), stimulated by intakes of carbohydrates and alcohol as shown in experimental studies [62, 64-67], and confirmed to some degree by InterAct findings: stronger correlations of evenchain SFA with intakes of potatoes, soft drinks, and alcohol than with direct dietary sources such as meat or dairy. Very little is currently known about the very-long chain SFA, and EPIC-InterAct provides impetus for further investigation of this group of SFA.

Taken together, EPIC-InterAct findings indicate that SFAs are not a single homogenous group, and recognise differences between the differential health effects of subtypes of blood
SFAs. The implication of this research is that it recognises that it may not be enough to provide public health messages about overall saturated fat intake, but that more nuanced messages acknowledging the food sources of different types of SFAs are required.

The biomarkers plasma vitamin C [68], 25-hydroxy vitamin D [57], and carotenoids are currently being measured in stored samples from EPIC-InterAct participants. The availability of a broad range of nutritional biomarkers in EPIC-InterAct will open up possibilities for more robust testing of causality of proposed diet-diabetes associations using genetic Mendelian randomisation experiments $[69,70]$. 


\section{The Association Between Physical Activity and the Risk of Type 2 Diabetes}

The physical activity questionnaire used in EPIC-Europe is relatively simple and includes questions that quantify physical activity at work and in recreation. Whilst originally it was intended that analysis could use the concepts of duration, intensity, and frequency of activities to provide a quantitative summary of total activity, initial research demonstrated that quantification was not valid [71]. This analysis also suggested that an ordered categorical variable that created a global index of physical activity for individuals without the attribution of specific estimates of energy expenditure was valid and repeatable. Subsequent work demonstrated that this index predicted other clinical endpoints such as cardiovascular disease, increasing its face validity. Within InterAct, a cross-country validation study was undertaken showing the validity of this four category summary index of activity in all 10 countries in EPIC [72]. In addition to demonstrating overall validity, this analysis also quantified the energy expenditure difference between physical activity categories, suggesting that each category difference was equivalent to a difference in physical activity energy expenditure of $380-460 \mathrm{~kJ}$ per day or $20 \mathrm{~min}$ of brisk walking. InterAct then went on to quantify the relationship between this summary index of activity and the incidence of diabetes, showing that for each category difference in the physical activity index, there was a $13 \%$ reduction in risk of type 2 diabetes in men and $7 \%$ in women [73•]. This quantification of the dose-response relationship is of value in guiding expectations about what can be achieved through small changes in population-level physical activity.

\section{Interaction Between Genes and Lifestyle Factors on the Risk of Type 2 Diabetes}

Through the studies described above, InterAct has, therefore, robustly characterised the relationship between the main lifestyle exposures and diabetes, which not only guides public health interventions, but also provides a platform for investigating how those associations are modified by genetic and developmental susceptibility. In the first such analysis, InterAct examined the association of a series of proven candidate genes for type 2 diabetes with disease risk examining interaction with lifestyle factors [74•]. The effect of the genetic score was significantly greater in younger individuals ( $p$-value for interaction $\left.=1.20 \times 10^{-4}\right)$. Relative genetic risk $($ per standard deviation; 4.4 risk alleles) was also larger in participants who were leaner, both in terms of body mass index ( $p$-value for interaction $\left.=1.50 \times 10^{-3}\right)$ and waist circumference $(p$-value for interaction $\left.=7.49 \times 10^{-9}\right)$. The analysis found no significant interactions between the genetic score, comprising these variants, and sex, diabetes family history, physical activity, or dietary habits assessed by a Mediterranean diet score. We concluded that the relative effect of this diabetes genetic risk score was greater in younger and leaner participants, but that because this sub-group is at low absolute risk, they would not be a logical target for preventive interventions. By contrast, we argued that the high absolute risk associated with obesity at any level of genetic risk highlighted the importance of universal rather than targeted approaches to lifestyle intervention. This conclusion is limited to the set of genetic variants that were examined in this particular analysis, and future work will focus on genomewide approaches and sets of genetic variants selected by specific biological hypotheses.

\section{Conclusions and Public Health Implications of the InterAct Study}

Beyond the specific conclusions of individual analyses that are summarised in this paper, the overall importance of the findings from the InterAct study is that it is creating a body of literature based on a large scale cohort across different European countries that forms a valid and robust foundation on which public health recommendations can be formed. In some instances, smaller studies have previously generated conflicting results and the strength of InterAct lies in the robustness of its conclusions. The development of additional layers of information, including objective nutritional biomarkers and genetics has opened up multiple lines of enquiry including the investigation of interaction and detailed examination of causal inference using genetic variants as instrumental variables. Such studies will not prove or disprove whether specific nutritional factors are causally linked to type 2 diabetes, but they can provide additional information which goes beyond the traditional observational epidemiological approach which is inherently limited by confounding. Whilst some have argued that the problems of confounding are only addressable by randomised controlled trials, the costs of undertaking trials of different preventive interventions are so prohibitive that there needs to be an investment into alternative approaches that can inexpensively provide additional information about causal interpretation, and thus provide greater confidence to public health recommendations. The structure established within the InterAct study provides a platform for such investigations in the future.

Acknowledgments We thank all the investigators of the InterAct project and all InterAct Consortium members for their contribution. Funding for the InterAct project was provided by the EU FP6 Programme (grant number LSHM_CT_2006_037197). The authors acknowledge support from the Medical Research Council Epidemiology Unit (grants MC_UU_12015/5 and MC_UU_12015/1). 


\section{Compliance with Ethics Guidelines}

Conflict of Interest Nita G. Forouhi and Nicholas J. Wareham declare that they have no conflict of interest.

Human and Animal Rights and Informed Consent This article does not contain any studies with animals performed by any of the authors. Ethical approval and individual informed consent was available for the EPIC study at each research centre.

Open Access This article is distributed under the terms of the Creative Commons Attribution License which permits any use, distribution, and reproduction in any medium, provided the original author(s) and the source are credited.

\section{References}

Papers of particular interest, published recently, have been highlighted as:

- Of importance

•- Of major importance

1. International Diabetes Federation. IDF Diabetes Atlas: 6th Editon. http://www.idf.org/diabetesatlas. Brussels, Belgium.: 2013. This report presents the most current estimates for the worldwide and regional burden of diabetes.

2. Gillies CL, Abrams KR, Lambert PC, et al. Pharmacological and lifestyle interventions to prevent or delay type 2 diabetes in people with impaired glucose tolerance: systematic review and meta-analysis. BMJ. 2007;334:299-307.

3. Knowler WC, Barrett-Connor E, Fowler SE, et al. Reduction in the incidence of type 2 diabetes with lifestyle intervention or metformin. N Engl J Med. 2002;346:393-403.

4. Ramachandran A, Snehalatha C, Mary S, Mukesh B, Bhaskar AD, Vijay V. The Indian Diabetes Prevention Programme shows that lifestyle modification and metformin prevent type 2 diabetes in Asian Indian subjects with impaired glucose tolerance (IDPP-1). Diabetologia. 2006;49:289-97.

5. Tuomilehto J, Lindstrom J, Eriksson JG, et al. Prevention of type 2 diabetes mellitus by changes in lifestyle among subjects with impaired glucose tolerance. N Engl J Med. 2001;344:1343-50.

6. Li G, Zhang P, Wang J, et al. The long-term effect of lifestyle interventions to prevent diabetes in the China Da Qing Diabetes Prevention Study: a 20-year follow-up study. Lancet. 2008;371:1783-9.

7. World Health Organization. Diet, nutrition and the prevention of chronic diseases. World Health Organisation Technical Report Series. Geneva: 2003.

8. Cornelis MC, Tchetgen EJ, Liang L, et al. Gene-environment interactions in genome-wide association studies: a comparative study of tests applied to empirical studies of type 2 diabetes. Am J Epidemiol. 2012;175:191-202.

9.• Langenberg C, Sharp S, Forouhi NG, et al. Design and cohort description of the InterAct Project: an examination of the interaction of genetic and lifestyle factors on the incidence of type 2 diabetes in the EPIC Study. Diabetologia. 2011;54:2272-82. This article provides important summary information about the design and conduct of the InterAct project.

10. Slimani N, Deharveng G, Unwin I, et al. The EPIC nutrient database project (ENDB): a first attempt to standardize nutrient databases across the 10 European countries participating in the EPIC study. Eur J Clin Nutr. 2007;61:1037-56.
11. Micha R, Wallace SK, Mozaffarian D. Red and processed meat consumption and risk of incident coronary heart disease, stroke, and diabetes mellitus: a systematic review and meta-analysis. Circulation. 2010;121:2271-83.

12. Aune D, Ursin G, Veierod MB. Meat consumption and the risk of type 2 diabetes: a systematic review and meta-analysis of cohort studies. Diabetologia. 2009;52:2277-87.

13. Pan A, Sun Q, Bernstein AM, et al. Red meat consumption and risk of type 2 diabetes: 3 cohorts of US adults and an updated metaanalysis. Am J Clin Nutr. 2011;94:1088-96.

14. Mannisto S, Kontto J, Kataja-Tuomola M, Albanes D, Virtamo J. High processed meat consumption is a risk factor of type 2 diabetes in the Alpha-Tocopherol, Beta-Carotene Cancer Prevention study. Br J Nutr. 2010;103:1817-22.

15. Lajous M, Tondeur L, Fagherazzi G, de Lauzon-Guillain B, Boutron-Ruaualt MC, Clavel-Chapelon F. Processed and unprocessed red meat consumption and incident type 2 diabetes among French women. Diabetes Care. 2012;35:128-30.

16. van Woudenbergh GJ, Kuijsten A, Tigcheler B, et al. Meat consumption and its association with C-reactive protein and incident type 2 diabetes: the Rotterdam Study. Diabetes Care. 2012;35:1499-505.

17. Bendinelli $\mathrm{B}$, on behalf of the InterAct Consortium. Association between dietary meat consumption and incident type 2 diabetes: the EPIC-InterAct study. Diabetologia. 2012;56:47-59.

18. Tong X, Dong JY, Wu ZW, Li W, Qin LQ. Dairy consumption and risk of type 2 diabetes mellitus: a meta-analysis of cohort studies. Eur J Clin Nutr. 2011;65:1027-31.

19. International Dairy Federation. Bulletin: The world dairy situation. 2007.

20. Hjartaker A, Lagiou A, Slimani N, et al. Consumption of dairy products in the European Prospective Investigation into Cancer and Nutrition (EPIC) cohort: data from 35955 24-hour dietary recalls in 10 European countries. Public Health Nutr. 2002;5:1259-71.

21. Sluijs I, Forouhi NG, Beulens JW, Consortium eaobotI. The amount and type of dairy product intake and incident type 2 diabetes: results from the EPIC-InterAct Study. Am J Clin Nutr. 2012;96:382-90.

22. Patel PS, Sharp SJ, Luben RN, et al. Association between type of dietary fish and seafood intake and the risk of incident type 2 diabetes: the European prospective investigation of cancer (EPIC)-Norfolk cohort study. Diabetes Care. 2009;32:1857-63.

23. Kaushik M, Mozaffarian D, Spiegelman D, Manson JE, Willett WC, Hu FB. Long-chain omega-3 fatty acids, fish intake, and the risk of type 2 diabetes mellitus. Am J Clin Nutr. 2009;90:613-20.

24. Zhou Y, Tian C, Jia C. Association of fish and n-3 fatty acid intake with the risk of type 2 diabetes: a meta-analysis of prospective studies. Br J Nutr. 2012;108:408-17.

25. Xun P, He K. Fish consumption and incidence of diabetes: metaanalysis of data from 438,000 individuals in 12 independent prospective cohorts with an average 11-year follow-up. Diabetes Care. 2012;35:930-8.

26. Patel PS, Forouhi NG, Kuijsten A, et al. The prospective association between total and type of fish intake and type 2 diabetes in 8 European countries: EPIC-InterAct Study. Am J Clin Nutr. 2012;95:1445-53.

27. Wallin A, Di Giuseppe D, Orsini N, Patel PS, Forouhi NG, Wolk A. Fish consumption, dietary long-chain n-3 fatty acids, and risk of type 2 diabetes: systematic review and meta-analysis of prospective studies. Diabetes Care. 2012;35:918-29. This meta-analysis highlights heterogeneity in the association of fish intake and type 2 diabetes by geographic location.

28. Carter P, Gray LJ, Troughton J, Khunti K, Davies MJ. Fruit and vegetable intake and incidence of type 2 diabetes mellitus: systematic review and meta-analysis. BMJ. 2010;341:c4229.

29. Cooper AJ, Forouhi NG, Ye Z, et al. Fruit and vegetable intake and type 2 diabetes: EPIC-InterAct prospective study and meta-analysis. Eur J Clin Nutr. 2012;66:1082-92. 
30. Malik VS, Popkin BM, Bray GA, Despres JP, Willett WC, Hu FB. Sugar-sweetened beverages and risk of metabolic syndrome and type 2 diabetes: a meta-analysis. Diabetes Care. 2010;33:2477-83. This article presents the first comprehensive systematic review and meta analysis opening up the debate about whether the habitual consumption of sugary drinks contributes to the development of future diabetes.

31. InterAct Consortium. Consumption of sweet beverages and type 2 diabetes incidence in European adults: results from EPIC-InterAct. Diabetologia. 2013;56:1520-30. This article increases our understanding of associations of both sugar sweetened and artificially sweetened beverages with incident diabetes, particularly paying attention to the influence of body mass index on the associations.

32. Malik VS, Popkin BM, Bray GA, Despres JP, Hu FB. Sugarsweetened beverages, obesity, type 2 diabetes mellitus, and cardiovascular disease risk. Circulation. 2010;121:1356-64.

33. InterAct Consortium. Tea consumption and incidence of type 2 diabetes in Europe: the EPIC-InterAct case-cohort study. PLoS ONE. 2012;7:e36910.

34. Alhazmi A, Stojanovski E, McEvoy M, Garg ML. The association between dietary patterns and type 2 diabetes: a systematic review and meta-analysis of cohort studies. J Hum Nutr Diet Off J Br Diet Assoc. 2014;27:251-60.

35. Romaguera-Bosch $\mathrm{D}$ et al. Mediterranean diet and type 2 diabetes risk in the European Prospective Investigation into Cancer and Nutrition (EPIC) study: the InterAct project. Diabetes Care. 2011;34:1913-8.

36. Hoffmann K, Schulze MB, Schienkiewitz A, Nothlings U, Boeing H. Application of a new statistical method to derive dietary patterns in nutritional epidemiology. Am J Epidemiol. 2004;159:935-44.

37. InterAct Consortium. Adherence to predefined dietary patterns and incident type 2 diabetes in European populations: EPIC-InterAct study. Diabetologia. 2014;57:321-33.

38. Wang ET, de Koning L, Kanaya AM. Higher protein intake is associated with diabetes risk in South Asian Indians: the Metabolic Syndrome and Atherosclerosis in South Asians Living in America (MASALA) study. J Am Coll Nutr. 2010;29:130-5.

39. Pounis GD, Tyrovolas S, Antonopoulou M, et al. Long-term animal-protein consumption is associated with an increased prevalence of diabetes among the elderly: the Mediterranean Islands (MEDIS) study. Diabetes Metab. 2010;36:484-90.

40. Tinker LF, Sarto GE, Howard BV, et al. Biomarker-calibrated dietary energy and protein intake associations with diabetes risk among postmenopausal women from the Women's Health Initiative. Am J Clin Nutr. 2011;94:1600-6.

41. Sluijs I, Beulens JW, van der A D, Spijkerman AM, Grobbee DE, van der Schouw YT. Dietary intake of total, animal, and vegetable protein and risk of type 2 diabetes in the European Prospective Investigation into Cancer and Nutrition (EPIC)-NL study. Diabetes Care. 2010;33:43-8.

42. van Nielen M, Feskens EJ, Mensink M, et al. Dietary protein intake and incidence of type 2 diabetes in Europe: the EPIC-InterAct casecohort study. Diabetes Care. 2014;37:1854-62.

43. Sluijs I, Beulens JW, van der Schouw YT, et al. Dietary glycemic index, glycemic load, and digestible carbohydrate intake are not associated with risk of type 2 diabetes in eight European countries. J Nutr. 2013;143:93-9.

44. Dong JY, Zhang L, Zhang YH, Qin LQ. Dietary glycaemic index and glycaemic load in relation to the risk of type 2 diabetes: a meta-analysis of prospective cohort studies. Br J Nutr. 2011;106:1649-54.

45. Liu S, Chou EL. Dietary glycemic load and type 2 diabetes: modeling the glucose-raising potential of carbohydrates for prevention. Am J Clin Nutr. 2010;92:675-7.

46. Barclay AW, Petocz P, Millan-Price J, et al. Glycemic index, glycemic load, and chronic disease risk-a meta-analysis of observational studies. Am J Clin Nutr. 2008;87:627-37.
47. Bhupathiraju SN, Tobias DK, Malik VS, et al. Glycemic index, glycemic load, and risk of type 2 diabetes: results from 3 large US cohorts and an updated meta-analysis. Am J Clin Nutr. 2014;100: 218-32.

48. van den Berg SW, van der A D, Spijkerman AM, et al. The association between dietary energy density and type 2 diabetes in Europe: results from the EPIC-InterAct study. PLoS ONE. 2013;8: e59947.

49. Wedick NM, Pan A, Cassidy A, et al. Dietary flavonoid intakes and risk of type 2 diabetes in US men and women. Am J Clin Nutr. 2012;95:925-33.

50. Song Y, Manson JE, Buring JE, Sesso HD, Liu S. Associations of dietary flavonoids with risk of type 2 diabetes, and markers of insulin resistance and systemic inflammation in women: a prospective study and cross-sectional analysis. J Am Coll Nutr. 2005;24: 376-84.

51. Knekt P, Kumpulainen J, Jarvinen R, et al. Flavonoid intake and risk of chronic diseases. Am J Clin Nutr. 2002;76:560-8.

52. Nanri A, Mizoue T, Takahashi Y, et al. Soy product and isoflavone intakes are associated with a lower risk of type 2 diabetes in overweight Japanese women. J Nutr. 2010;140:580-6.

53. Nettleton JA, Harnack LJ, Scrafford CG, Mink PJ, Barraj LM, Jacobs Jr DR. Dietary flavonoids and flavonoid-rich foods are not associated with risk of type 2 diabetes in postmenopausal women. $\mathrm{J}$ Nutr. 2006;136:3039-45.

54. Mueller NT, Odegaard AO, Gross MD, et al. Soy intake and risk of type 2 diabetes in Chinese Singaporeans. Eur J Nutr. 2012;51: 1033-40.

55. Zamora-Ros R, Forouhi NG, Sharp SJ, et al. The association between dietary flavonoid and lignan intakes and incident type 2 diabetes in European populations: the EPIC-InterAct study. Diabetes Care. 2013;36:3961-70.

56. Zamora-Ros R, Forouhi NG, Sharp SJ, et al. Dietary intakes of individual flavanols and flavonols are inversely associated with incident type 2 diabetes in European populations. J Nutr. 2013; 144:335-43.

57. Forouhi NG, Ye Z, Rickard AP, et al. Circulating 25hydroxyvitamin $\mathrm{D}$ concentration and the risk of type 2 diabetes: results from the European Prospective Investigation into Cancer (EPIC)-Norfolk cohort and updated meta-analysis of prospective studies. Diabetologia. 2012;55:2173-82.

58. Wang LY, Summerhill K, Rodriguez-Canas C, et al. Development and validation of a robust automated analysis of plasma phospholipid fatty acids for metabolic phenotyping of large epidemiological studies. Genome Med. 2013;5:39.

59. Forouhi NG, Koulman A, Sharp SJ, et al. Differences in the prospective association between individual plasma phospholipid saturated fatty acids and incident type 2 diabetes: the EPIC-InterAct case-cohort study. Lancet Diabetes Endocrinol. 2014. doi:10.1016/S2213-8587(14)70146-9. This article highlights that different individual saturated fatty acids are heterogeneous in associations with incident type 2 diabetes including some with postive and some with inverse associations, highlighting the need to gain a deeper insight into the role of dietary versus endogenous influences on circualting saturated fatty acids.

60. Smedman AE, Gustafsson IB, Berglund LG, Vessby BO. Pentadecanoic acid in serum as a marker for intake of milk fat: relations between intake of milk fat and metabolic risk factors. Am J Clin Nutr. 1999;69:22-9.

61. Wolk A, Vessby B, Ljung H, Barrefors P. Evaluation of a biological marker of dairy fat intake. Am J Clin Nutr. 1998;68:291-5.

62. Hodson L, Skeaff CM, Fielding BA. Fatty acid composition of adipose tissue and blood in humans and its use as a biomarker of dietary intake. Prog Lipid Res. 2008;47:348-80. 
63. Aune D, Norat T, Romundstad P, Vatten LJ. Dairy products and the risk of type 2 diabetes: a systematic review and dose-response metaanalysis of cohort studies. Am J Clin Nutr. 2013. This article presents a comprehensive review of the assocations between types of dairy products and risk of diabetes.

64. Hudgins LC, Hellerstein M, Seidman C, Neese R, Diakun J, Hirsch J. Human fatty acid synthesis is stimulated by a eucaloric low fat, high carbohydrate diet. J Clin Invest. 1996;97:2081-91.

65. King IB, Lemaitre RN, Kestin M. Effect of a low-fat diet on fatty acid composition in red cells, plasma phospholipids, and cholesterol esters: investigation of a biomarker of total fat intake. Am J Clin Nutr. 2006;83:227-36.

66. Siler SQ, Neese RA, Hellerstein MK. De novo lipogenesis, lipid kinetics, and whole-body lipid balances in humans after acute alcohol consumption. Am J Clin Nutr. 1999;70:928-36.

67. Hellerstein MK, Schwarz JM, Neese RA. Regulation of hepatic de novo lipogenesis in humans. Annu Rev Nutr. 1996;16:523-57.

68. Harding $\mathrm{AH}$, Wareham NJ, Bingham SA, et al. Plasma vitamin C level, fruit and vegetable consumption, and the risk of new-onset type 2 diabetes mellitus: the European prospective investigation of cancer-Norfolk prospective study. Arch Intern Med. 2008;168: 1493-9.

69. Lawlor DA, Harbord RM, Sterne JA, Timpson N, Davey SG. Mendelian randomization: using genes as instruments for making causal inferences in epidemiology. Stat Med. 2008;27:1133-63.

70. Davey SG. Use of genetic markers and gene-diet interactions for interrogating population-level causal influences of diet on health. Genes Nutr. 2011;6:27-43.

71. Wareham NJ, Jakes RW, Rennie KL, et al. Validity and repeatability of a simple index derived from the short physical activity questionnaire used in the European Prospective Investigation into Cancer and Nutrition (EPIC) study. Public Health Nutr. 2003;6:407-13.

72. InterAct Consortium, Peters $\mathrm{T}$, Brage $\mathrm{S}$, et al. Validity of a short questionnaire to assess physical activity in 10 European countries. Eur J Epidemiol. 2012;27:15-25.

73. InterAct Consortium, Ekelund U, Palla L, et al. Physical activity reduces the risk of incident type 2 diabetes in general and in abdominally lean and obese men and women: the EPIC-InterAct Study. Diabetologia. 2012;55:1944-52. This article provides an evidence base among a large and heterogeneous European population of the potential benefit of physical activity for the prevention of type 2 diabetes.

74. Langenberg C, Sharp SJ, Franks PW, et al. Gene-lifestyle interaction and type 2 diabetes: the EPIC interact case-cohort study. PLoS Med. 2014;11:e1001647. This article examines the interaction between genetic and several lifestyle factors and diabetes risk factors on the risk of type 2 diabetes. 